\title{
Test Site Name
}

National Cancer Institute

\section{Source}

National Cancer Institute. Test Site Name. NCI Thesaurus. Code C90472.

The name of the location in which a test is managed. 